\title{
Foot pressure measurement system and early detection of joint issues
}

\author{
Abhishek Kenchgundi", Stuti Shah and Dinesh R. Damodar \\ Government Engineering College, Gandhinagar, India
}

Received: 15-September-2019; Revised: 20-October-2019; Accepted: 24-October-2019

(C)2019 Abhishek Kenchgundi et al. This is an open access article distributed under the Creative Commons Attribution (CC BY) License, which permits unrestricted use, distribution, and reproduction in any medium, provided the original work is properly cited.

\begin{abstract}
To determine foot plantar pressure during the day to day activities and real-time analysis, one needs a compact system capable of measuring foot pressure and providing accurate results. This project presents a low cost, reliable, compact foot plantar pressure acquisition system which we are developing. Strengths and boundaries of present-day structures are discussed and a foot plantar pressure device is suitable for measuring excessive stress distributions under the foot with excessive accuracy and reliability. This device is primarily based on highly linear strain sensors without a hysteresis. Various applications would need a different number of sensors spread over inside the shoe-sole. In this work, our investigation is restricted to simple applications like measuring the foot pressure when the person is standing still, or the speed when the person is moving, or whether she/he is escalating up or down the stairs. Our Project provides unique insight on foot function, helping clinicians conduct more complete assessments and objectively evaluate treatments. It includes a small sensor module that determines the foot pressure distribution in actual time that allows us to visualize and analyze the information. At the end of the experiment, real-time results in the form of intensity graph and graphical data of pressure point with a particular weigh have been achieved. A reference study of this data with the ideal situation will lead us to the early detection of joint issues.
\end{abstract}

\section{Keywords}

Foot pressure measurement, Force sensing resistor, LabVIEW GUI, Universal size footsole.

\section{Introduction}

The anatomical structure of the human body has evolved since the beginning of the era. Human locomotion and walking pattern of the human have a significant impact on the health of the entire skeleton structure of the body. Inappropriate and non-uniform distribution of the pressure on the foot is the house of long-term arthritis and dislocation of the knee, pelvis, spine. This conception has been authenticated by the various research studies, that excess non-uniform distribution on any part of the foot will create some undesired outcomes and unwanted consequences for the subject. The measurement of the same pressure is useful for the determination of the gait cycle. It is also identified that age-related anatomical and physiological changes in foot bone and ligament structure affect the foot pressure distribution during gait $[1,2]$.

\footnotetext{
*Author for correspondence
}

In elder subjects, weight-bearing capacity on the lateral side of the foot during the heel touch and toeoff phases may affect stability during walking [2]. The main objective of this study is to learn the average data of the foot pressure by inserting the sole inside the shoes. This data will be monitored by the controller algorithm. The controller compares the actual average reading with data feed to it with properly managed abnormal reference data.

Feet provide the primary surface of interaction with the environment during locomotion. Thus, it is important to diagnose problems associated with the feet at the early stage to prevent injury, management of risk associated with feet. So, it is essential to examine foot plantar characteristics. Concerning applications involving disease diagnosis, many researchers have focused on foot ulceration problems due to diabetes that can result in excessive foot plantar pressures in particular areas under the foot. Hence accurate and reliable foot plantar pressure measurement devices must be developed. 
Arthritis is also known as joint inflammation. It is being predicted that almost $40 \%$ of the men and $47 \%$ of the woman will suffer from arthritis during their lifetime [3]. While knee dislocation is observed in sportspersons and requires more than 7 months of the recovery period to regain full range of motion. It involves wear and tear of Anterior cruciate ligament, posterior cruciate ligament and medial collateral ligament [4]. Neglecting early-stage symptoms of the knee dislocation and arthritis could have a worse impact on the spine and pelvis, in some cases, it could be a situation might translate from osteoarthritis to spine dislocation.

The development of compact, lightweight, and low energy consuming circuit solutions for healthcare sensor applications is raising important research focus given the rapid growth and technological advancement in healthcare monitoring devices, microfabrication processes, and wireless communication. One vicinity is in the call for employing researchers in biomedical and sportsrelated applications is the analysis of foot plantar stress distributions to determine the interface stress among the foot plantar surface and the shoe sole. Typical applications are custom designing of footwear, analysis of sports performance and prevent injury, improvement in balance control, and diagnosing disease. More recently innovative applications have also emerged to human identification, biometric, allocation of monitoring posture and rehabilitation support systems. With the determination of the foot pressure by sensory output, we can identify what problem can cause the subject shortly with his walking pattern. We can identify precautionary steps to stop those side effects by changing our gait cycle with proper consciousness. With this diagnosis, we can predict all the diseases of the skeleton system that the user might get a victim of. It involves arthritis, knee dislocation, pelvic dislocation, spine dislocation.

\section{Research methodology}

In this project, the process used to measure pressure from the prime points of the foot can be done by using various types of sensors based on various technologies such as capacitive sensors, resistive sensors, piezoelectric sensors, and piezoresistive sensors. These sensors convert the pressure measured in the proportion value of voltage or current. For the better performance of the sensor, key specifications must include hysteresis, sensing size, linearity, pressure range, temperature sensitivity. Pressure sensors can be classified as resistive sensors, capacitive sensors, piezoelectric sensors, and piezoresistive sensors. In this project, we are using resistive sensors namely Force Sensitive Resistors (FSR) which convert the pressure detected from the foot surface of the sensor to the resistance. Figure 1 represents the block diagram for the types of foot strikes.

Early determination of joint diseases

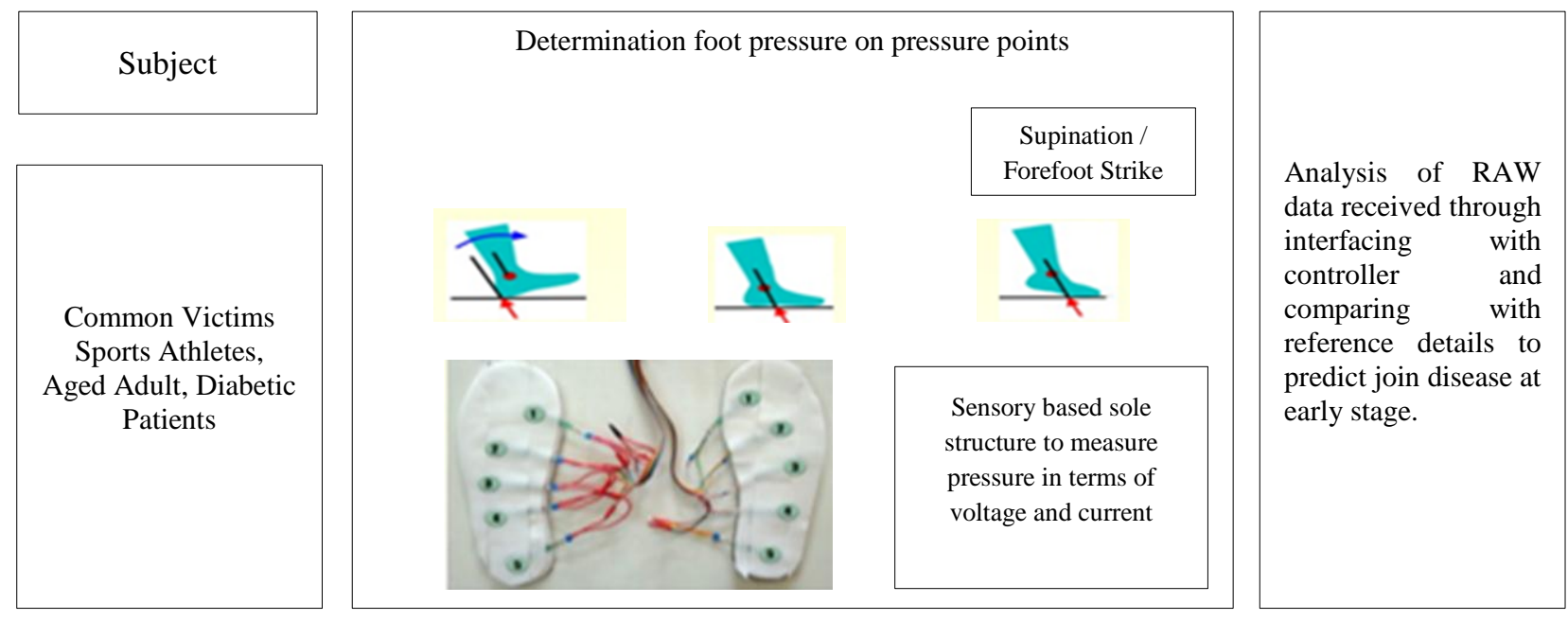

Figure 1 Block diagram for the types of foot strikes 
The maximum unusual type of foot strike is the heel strike and it takes place whilst someone lands on their heel first, now and then slapping their foot on the floor even as jogging. Midfoot strike, which is considered a preferred form of foot strike, is when an individual land with a flat foot. A forefoot strike or Toe strike, which is taken into consideration to be excellent, happens is while the individual lands on the ball of the foot in the direction of his/her feet. The ideal way of walking involves the midfoot and forefoot strike, as opposed to the heel strike.

\subsection{Design methodology}

Determination of foot pressure requires a proper identification method of pressure points on the foot. Pressure points in the foot are those that get continuously involved in the walking pattern and gait cycle. When placing a foot on the surface, pressure occupies on the foot and got distribute based upon the shape and its position on the surface along with its geometrical details.

In order to make pressure measurements on the foot for diagnosis purposes, there are 10 pressure points are considered as very ideal points which have been illustrated in Figure 2. As it can be seen from the Figure 1 that there are 10 main points on the feet where peer pressure on the feet and according to that we have decided to detect and measure pressure from 5 major points of the feet that holds the maximum pressure on it and as per the reflexology of the foot of different size we have considered to make the foot sole of 3 varying sizes of US 5, US 8, US 11.

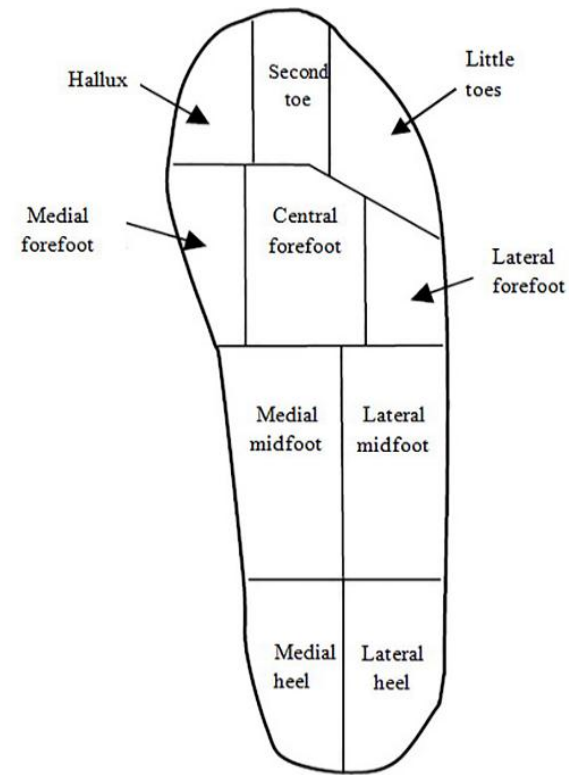

Figure 2 Ideal pressure points at foot planar [5]
Figure 3 shows the single FSR placed foot insole (first design Prototype). Figure 4 shows the second design prototype for universal sole. Figure 5 shows the third design prototype for universal sole.
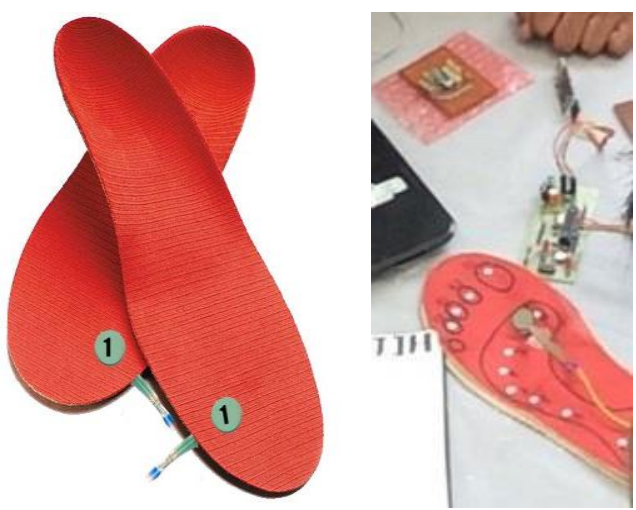

Figure 3 Single FSR placed foot insole (First design prototype)

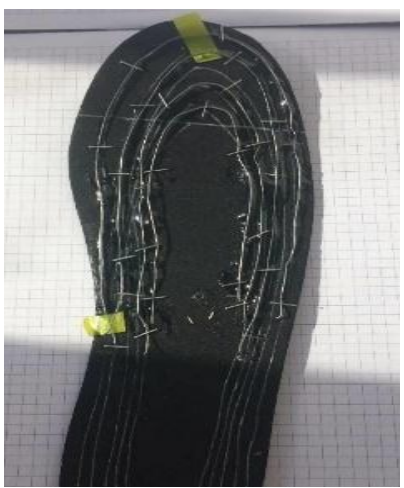

Figure 4 Second design prototype for universal sole

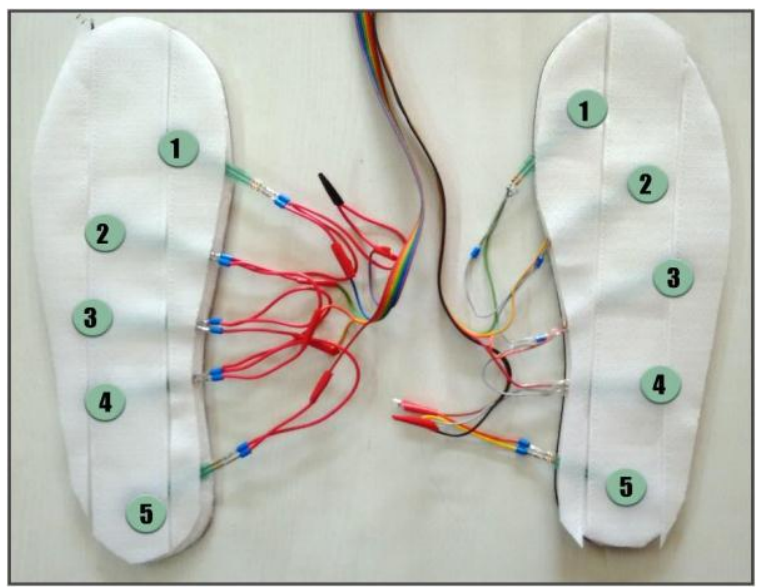

Figure 5 Third design prototype for universal sole

With the foot sole design of the single sensor and its interfacing, then emphasizes the requirement of universal sole instead of traditional one size sole. To 
overcome this, we designed a whole sole made out of Velcro, which solved our problem of getting the variable output with fewer errors. The soft side of the Velcro was kept in the sole and sensors were placed between the Velcro so that it was free from any kind of pressure or shock until we apply for it. This way we could place the sensor on the key points covering the important area of the foot.

The second design, prototype was consisted of the sole structure, 3 wires with different size deposited on the sole aiming to visually recognize the size of the sole and adjust the sensory part according to that, but not convenient due to the itchy structuring of wire. Moving forward to the third prototype which has overcome this problem by replacing that wired structure with Velcro pad on the surface. With this design, we have amended this solely with the functionality to wear by all users even with the different sizes of foot. Figure 6 shows the final design of the prototype. Figure 7 shows the hardware flowchart.

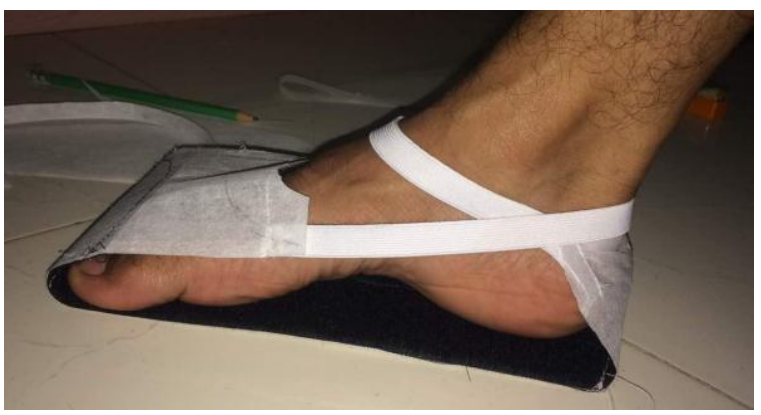

Figure 6 Final design of the prototype

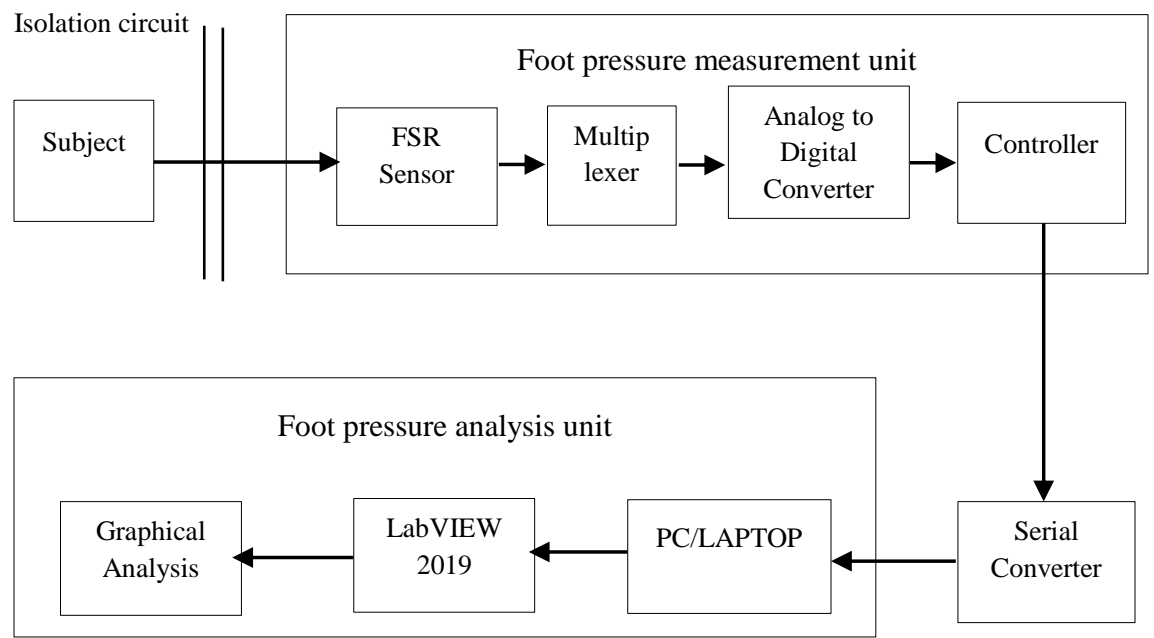

Figure 7 Hardware flowchart

\subsection{Hardware methodology}

As shown in the block diagram, the signal is acquired from the foot through the sensors. The signal sensed by the sensor is filtered by using analog filters and the signal is amplified with respect to the gain required. Further, the microcontroller is required to process the data as per our needs. So, to initiate a microcontroller we tend to provide power supply to it. As the signal is processed the output from the microcontroller is sent to Serial Converter through which the output of the system can be viewed on a computer. We have used only 10 FSR sensors to measure foot pressure. So, for the measurement of the pressure generated on both limbs can be detected by placing sensors beneath both limbs.
FSRs are resistors (Figure 8) that changes its resistive value (in ohms $\Omega$ ) depending on how much it is pressed [6].

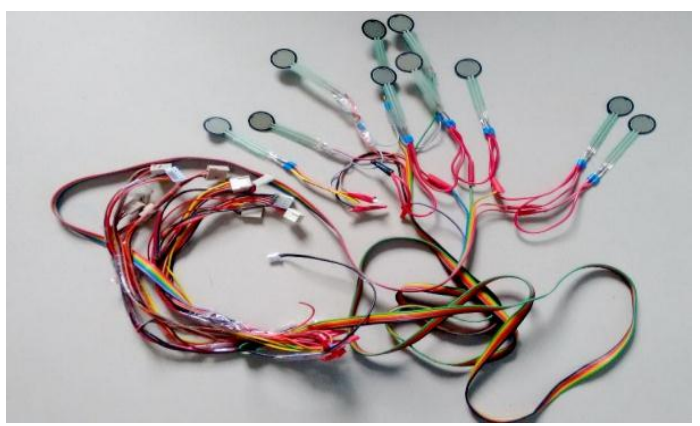

Figure 8 Force sensitive resistors 
The resistance of the FSR changes when pressure applied to it (Table 1). Resistance remains high when no pressure is applied, and drop down exponentially after the force applied. In addition, the multiplexer circuit has been employed here to cascade and unite the output of all the sensors as well as send it to the controller. This requirement arose due to the ability of controlling constraint to the 8 sensors only, while 10 sensors are a minimal requirement for our purpose. The controller collects all the raw data of the sensory input and pushes the same to the serial converter which translates voltage levels of the input in the format that could be understood by the laptop/computer. The main concern behind this translation is to use LabVIEW graphical \& visual tools to get an actual comparison on foot, which requires a lot of more complicated calculations if calculated manually or even with the controller algorithms.

Table 1 Resistance changes of FSR sensor when pressure applied

\begin{tabular}{cccccc}
\hline $\begin{array}{c}\text { Force } \\
(\mathbf{l b})\end{array}$ & $\begin{array}{c}\text { Force } \\
(\mathbf{N})\end{array}$ & $\begin{array}{c}\text { FSR Resistance } \\
(\mathbf{K} \boldsymbol{\Omega})\end{array}$ & $\begin{array}{c}(\mathbf{F S R}+\mathbf{R}) \\
(\mathbf{K} \boldsymbol{\Omega})\end{array}$ & $\begin{array}{c}\text { Current through } \\
\text { FSR + R (mA) }\end{array}$ & $\begin{array}{c}\text { Voltage across R } \\
(\mathbf{V})\end{array}$ \\
\hline None & None & Infinite & Infinite & 0 & 0 \\
\hline $0.04 \mathrm{lb}$ & 0.2 & 30 & 40 & 0.13 & 1.3 \\
\hline $0.22 \mathrm{lb}$ & 1 & 6 & 16 & 0.31 & 3.1 \\
\hline $2.2 \mathrm{lb}$ & 10 & 1 & 11 & 0.45 & 4.5 \\
\hline $22 \mathrm{lb}$ & 200 & 0.25 & 10.25 & 0.49 & 4.9 \\
\hline
\end{tabular}

\section{Result and discussion}

The LabVIEW GUI interpretation method has proved useful in our fulfilment of the goal. LabVIEW 2019 software running on windows 10 operating system interfaced with the controller. This topology served a basic concept as controller sends data through TX pins and receives an acknowledgement via RX pins. PC/Laptop sends data through the TX pins of the USB and receive data through the RX pins. LabVIEW DAQ, and SCXI have employed here to acquire the real time output.
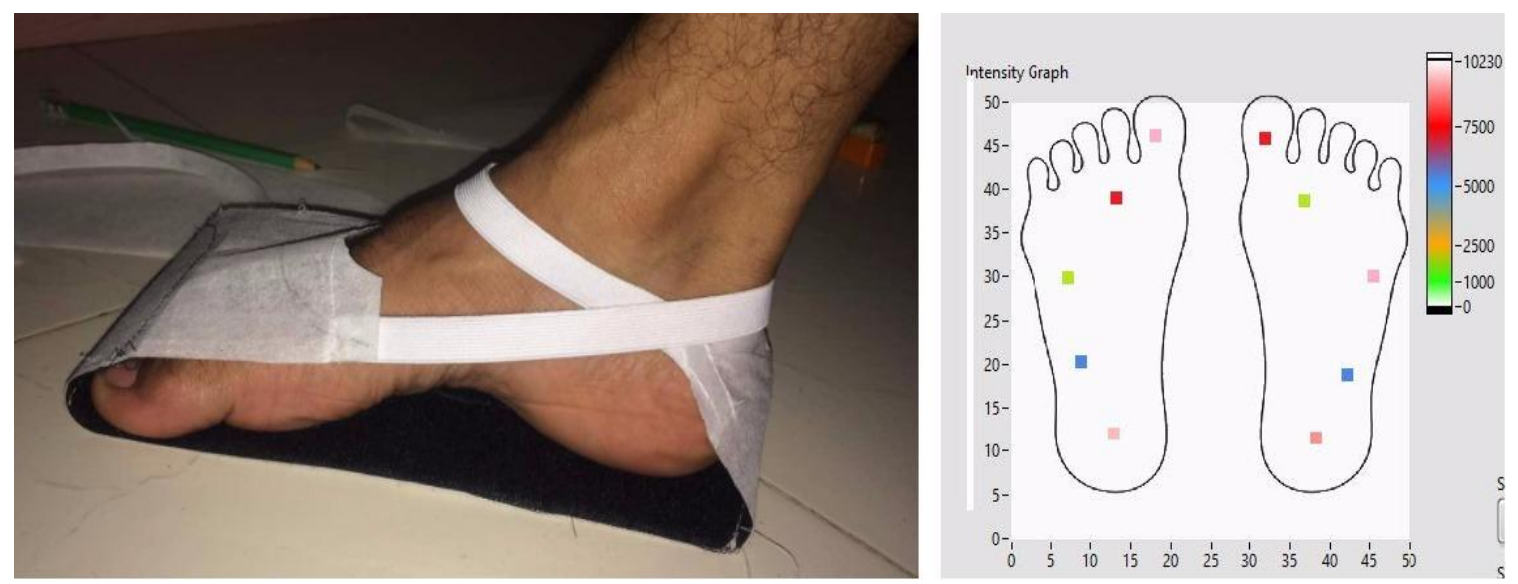

Figure 9 Intensity graph of foot pressure measurement
As portrayed in the results of the intensity graph, as we put the foot on the sole under which FSR sensors are facilitated, a force distribution will occur as a result. This force distribution may vary from the uniform to non-uniform. This outcome needs to compare with the ideal data of a normal person who is preciously healthy and walks/run with ideal force distribution on foot. As plotted in Figure 10. Sensor 1 to 5 are represented here on the left foot and sensor 6 to 10 are represented here on the right foot. Figure 9 reveals a color intensity that varies with the pressure applied to the foot. Excess implementation of weight on any portion of the foot will cause a long-term worst impact on the joint. 


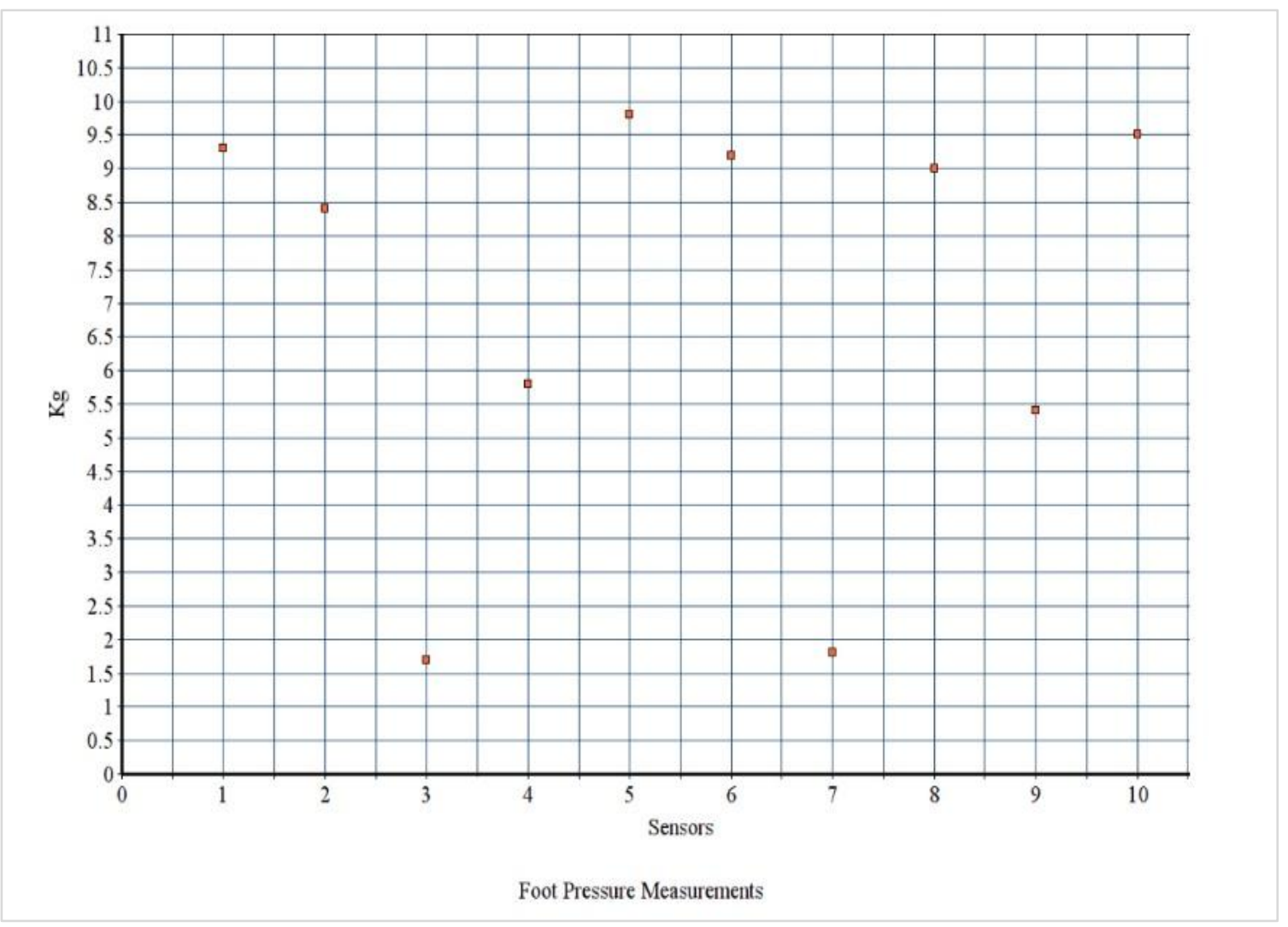

Figure 10 Plotted details of intensity graph

\section{Conclusion and future work}

This system will help in a clinical setting which used by podiatrists, orthotics, prosthetics, and physical therapists around the world and also to make a suitable sole to diagnose the problem claiming related to foot plantar. The future scope of this project is clear that various techniques capable of accurate, reliable and effectively efficient measurement of foot plantar pressure are essential to be developed. The same device will be helpful and useful to the giant shoe company with the interference with the low energy Bluetooth and lucrative display screen in order to instantly determine the walking/running walking pattern/running pattern of the user. Once it determined, the company can build custom shoes that will increase the mobility of the use and minimize the chances of a joint issue in the future.

\section{Acknowledgment}

Faculty of government engineering college has helped us in developing this prototype. We are grateful and thankful to the Ghanshyam D. Parmar as he has mentored us for the preparation of this project.

\section{Conflicts of interest}

The authors have no conflicts of interest to declare.

\section{References}

[1] Rodgers MM. Dynamic foot biomechanics. Journal of Orthopaedic \& Sports Physical Therapy. 1995; 21(6):306-16.

[2] Hessert MJ, Vyas M, Leach J, Hu K, Lipsitz LA, Novak V. Foot pressure distribution during walking in young and old adults. BMC Geriatrics. 2005; 5(8):1-8.

[3] Senthelal S, Thomas MA. Arthritis. In StatPearls [Internet] 2019. Stat Pearls Publishing.

[4] Henrichs A. A review of knee dislocations. Journal of Athletic Training. 2004; 39(4):365-9.

[5] Teymouri M, Halabchi F, Mirshahi M, Mansournia MA, Ahranjani AM, Sadeghi A. Comparison of plantar pressure distribution between three different shoes and three common movements in futsal. PLOS One. 2017 ; 12(10).

[6] https://www.digikey.in/en/producthighlight/i/interlink/fsr-400-round-force-sensingresistor? utm_adgroup=Resistors\&utm. Accessed 20 August 2019. 


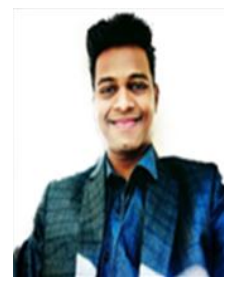

Abhishek Kenchgundi is a Biomedical Engineer, born and brought up in Ahmedabad, Gujarat, India. He completed his bechelor's degree in Biomedical Engineering in 2017 from Gujarat Technological University at the Government Engineering College, Gandhinagar from India. His Area of Interest is in Neuroscience, Bio-mechanics, Medical Imaging, Image guiding surgeries. $\mathrm{He}$ served as a coordinator and core team member in various international and national conferences, workshops and seminars. Currently he is employed as a Product Specialist (Biomedical Engineer) at Hester Diagnostics Pvt. Ltd. for Neurovascular products.

Email: abhiken1994@gmail.com

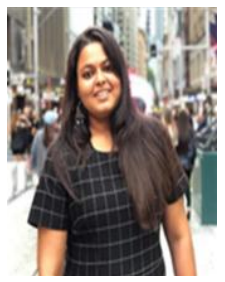

Stuti Kalpesh Shah is a Biomedical Engineer, born and brought up in Ahmedabad, Gujarat India. She did her Bachelors of Engineering, major in Biomedical Engineering in year 2017. Her area of interest is in Biomedical Instrumentation, Human Anatomy as well as in the field of Management so currently she is a PG student in the course named "Masters in Biomedical Engineering and Management" at University of Technology Sydney, Australia.

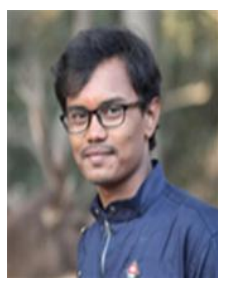

Dinesh R. Damodar currently persuing his skills in research as a independent researcher and self-employed. He has pursued his bachelors in Biomedical Engineering from Gujarat Technological University particularly from Government Engineering College. $\mathrm{He}$ is aspiring towards healthcare industry innovation. Major area of interest are Rehabilitaion Instrumentaton, Neuroscience, Biomechanics, Biomedical Instrumenation, Artificial Intelligence, Robotics, Machine learning. 